\title{
Restricted access or access all areas?: a new cadherin- like protein upregulated in the inflamed esophagus
}

\author{
DA Knight ${ }^{1-4}$ and PM Hansbro ${ }^{1,2}$
}

Like all epithelial tissues, the intestinal epithelium functions as a physical barrier and forms the front line of mucosal immunity. This multitasking ability is enabled by the structural organization of the epithelium. In the gastrointestinal tract, the correct functioning of the intestinal epithelial barrier (IEB) is crucial to ensure gastrointestinal health. ${ }^{1}$ Breaches of the IEB are associated with a myriad of diseases, including ulcerative colitis, Crohn's disease (CD), eosinophilic gastritis (EG), and eosinophilic esophagitis (EoE). ${ }^{2}$

The integrity of the IEB is maintained by the multiple epithelial junctional complexes that tether epithelial cells to one another and to the underlying extracellular matrix ${ }^{3}$ (Figure 1). Basal cells attach to the basal lamina via hemidesmosomes, whereas cell-cell contacts called desmosomes provide structural attachments between basal cells and columnar cells. Gap junctions provide direct accessibility as portholes between adjacent cells, whereas tight junctions regulate paracellular permeability and the passage of solutes and ions between cells. Tight junctions form the apical-most complex and are made up of transmembrane proteins such as the junctional adhesion molecules, occludins, and claudins, which anchor to the cytoskeleton via zonula occludens (ZO)1-3 and cingulin. Adherens junctions (AJs), together with desmosomes, provide the necessary adhesive forces to maintain correct cell-cell interactions. The transmembrane component of AJs, epithelial cadherin (E-cadherin; $\mathrm{CDH} 1$ ), is also an important modulator of cell signaling through its interactions with cytoplasmic binding partners, $\alpha$ and $\beta$-catenins, as well as several growth factor receptors.

In contrast to the role of tight junctions in epithelial structure and function, much remains to be discovered about $\mathrm{AJ}$ structure and function in the normal gut, and how they contribute to homeostasis of the IEB. In the September 2017 issue of Mucosal Immunology, Caldwell and colleagues ${ }^{4}$ described the structure, function, and binding partners of a novel cadherin-like protein, called $\mathrm{CDH} 26$, on gastric and esophageal epithelial cells of patients with EG and EoE. The authors showed that $\mathrm{CDH} 26$ shares overlapping structure and functions with other epithelial cadherins, comprising five extracellular cadherin repeats in the putative extracellular portion of the protein, a predicted transmembrane domain, a C-terminal cytoplasmic region, and strong binding to the classic alpha- and beta-catenins as well as p120 catenin. Ligation of CDH26 also induces calciumdependent cell-cell adhesion.

Inflammation of and damage to the mucosal epithelium is associated with significant disruption of interepithelial junctions and increased epithelial permeability. In other mucosal epithelia, such as the airways of asthmatics, expression of adhesion proteins is reduced, and this is compounded following exposure to noxious agents. ${ }^{5,6}$ One of the most novel findings of Caldwell and colleagues' study was that in both EG and EoE, the vast majority of $\mathrm{CDH} 26$ expression was observed on inflamed epithelial cells. In contrast, no significant change in $\mathrm{CDH} 26$ expression was seen in patients with $\mathrm{Hel}$ icobacter pylori gastritis compared with controls, suggesting that the upregulation is confined to allergic inflammation. This would make CDH26 unique, as the only cadherin family member whose expression is significantly increased in mucosal tissue in allergic inflammation.

Like all good studies, this one raises more questions. What are the underlying mechanism(s) that regulate $\mathrm{CDH} 26$ expression? How early in disease progression does the increased expression occur? Why would an adhesion molecule that is virtually absent in noninflamed conditions be so dramatically upregulated in allergic inflammation? The answer to the last question may be related to earlier findings that $\mathrm{CDH} 1$ binds to the integrin $\alpha E \beta 7$ (CD103) on lymphocytes and regulates the activation and localization of epidermal and intestinal intraepithelial lymphocytes. ${ }^{7}$ Caldwell and colleagues found that $\mathrm{CDH} 26$ interacts with not only integrin $\alpha 4$ but also $\alpha \mathrm{E}$ and as such might function like $\mathrm{CDH} 1$ to regulate localization or activation of leukocytes

\footnotetext{
${ }^{1}$ School of Biomedical Sciences and Pharmacy, University of Newcastle, Callaghan, New South Wales, Australia; ${ }^{2}$ Priority Research Centre for Healthy Lungs, Hunter Medical Research Institute, University of Newcastle, New Lambton Heights, New South Wales, Australia; ${ }^{3}$ Department of Anesthesiology, Pharmacology, and Therapeutics, University of British Columbia, Vancouver, British Columbia, Canada; ${ }^{4}$ School of Medicine and Pharmacology, University of Western Australia, Perth, Western Australia, Australia
} 


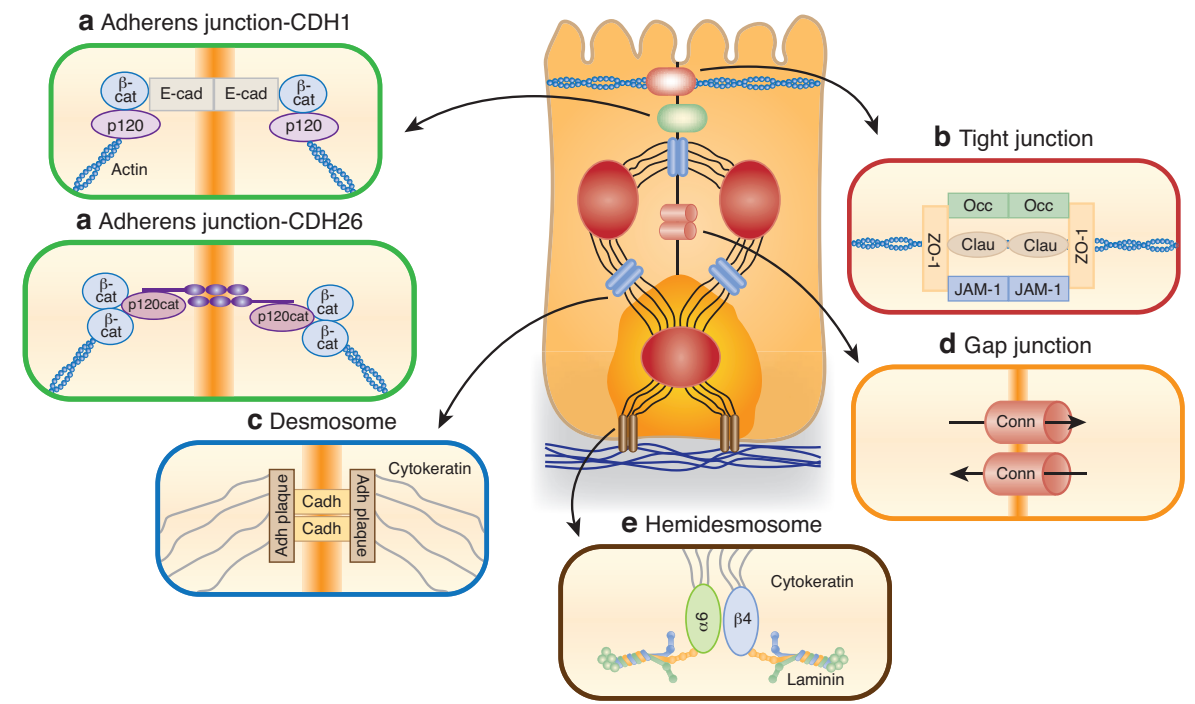

Figure 1 (a) Adherens junctions are composed of several proteins (E-cad, E-cadherin; $\beta$-cat, $\beta$-catenin; p120, p120 catenin) that attach to the actin cytoskeleton (yellow). (b) Tight junctions include proteins such as occludin (Occ), claudin (Clau), and junctional adhesion molecule 1 (JAM-1) held together by the scaffold zonula occludens-1 (ZO-1), which is tethered to the actin cytoskeleton (yellow). (c) Desmosomes attach neighboring cells via adhesion plaques (Adh plaque) composed of cadherins (Cadh), desmoplakin, and desmoglein tethered to the intermediate filament cytokeratins. (d) Gap junctions are made of connexins (Conn) that allow solute transport directly between cells. (e) Hemidesmosomes attach basal cells to the basement membrane using $\alpha 6 \beta 4$ integrins, which attach intracellular cytokeratin filaments to extracellular laminin.

during allergic responses. ${ }^{4}$ While $\mathrm{CDH} 26$ might be involved in promoting disease, the authors speculate that upregulation of $\mathrm{CDH} 26$ could, alternatively, be part of the resolution phase, promoting the return of epithelial homeostasis. They suggest that CDH26 activation may inhibit particular subsets of $\mathrm{CD}^{+} \mathrm{T}$ cells, for example, regulatory $\mathrm{T}$ cells, which are known to be increased in EoE or more select CD4+ T-cell subsets. This is an interesting hypothesis, particularly in light of the recent findings by Lamb and colleagues ${ }^{8}$ describing a population of colonic proinflammatory $\mathrm{CD} 4^{+} \alpha \mathrm{E} \beta 7^{+} \mathrm{T}$ cells that are enriched for a T-helper 17 (Th17) profile and play a role in the pathobiology of ulcerative colitis. It would be interesting to determine whether $\mathrm{CDH} 26$ recruits or suppresses activation of these cells in the esophagus. Thus, depending on the subset of T cells inhibited, the molecule could serve to either dampen or accelerate Th2-associated inflammatory responses. This would be a crucial next piece to the puzzle.
How, then, does the study by Caldwell et al. fit in with the current knowledge of strategies to improve gastrointestinal mucosal wound healing? The authors had previously reported that $\mathrm{CDH} 26 \mathrm{mRNA}$ was only minimally reduced by glucocorticoid treatment in esophageal biopsies of EoE patients and that expression still remained significantly higher than that in control subjects. ${ }^{9}$ While uncovering the roles of $\mathrm{CDH} 26$, Caldwell and colleagues obtained evidence that the protein can be exploited to generate a potential therapeutic. Specifically, they generated a fusion protein of $\mathrm{CDH} 26-\mathrm{Fc}$ (and CDH1$\mathrm{Fc}$ ) and showed that it dose-dependently inhibited the increase in $\mathrm{CD} 25^{+}$cells and interleukin-2 in cells subjected to T-cellreceptor stimulation. Based on this evidence, the authors suggest that $\mathrm{CDH} 26$ is immunosuppressive, at least in vitro. Thus, targeting AJs to facilitate IEB function would be an attractive treatment concept, to conventional therapy. It would also be of significant interest to examine whether particularly in patients who are refractory activation or inhibition affects other prohealing properties of this molecule.

The epithelium is continually exposed to environmental factors that induce stress and, through coordinated innate immune, barrier, and wound-healing functions, normally copes with these stressors appropriately. Given that mucosal healing is associated with improved patient outcomes, ${ }^{10}$ a much more comprehensive understanding of IEB function and dysfunction will undoubtedly provide key data for improving both the clinical management of patients and identification and design of novel therapies.

\section{ACKNOWLEDGMENTS}

The authors sincerely thank $\mathrm{Dr}$ Stephanie Warner for her artwork in Figure 1.

\section{DISCLOSURE}

The authors declared no conflict of interest.

(C) 2018 Society for Mucosal Immunology

\section{REFERENCES}

1. Odenwald, M.A. \& Turner, J.R. The intestinal epithelial barrier: a therapeutic target? Nat. Rev. Gastroenterol. Hepatol. 14, 9-21 (2017).

2. Schulzke, J.D. et al. Epithelial tight junctions in intestinal inflammation. Ann. N.Y. Acad. Sci. 1165, 294-300 (2009).

3. Watson, A.J. \& Hughes, K.R. TNF- $\alpha$-induced intestinal epithelial cell shedding: implications for intestinal barrier function. Ann. N.Y. Acad. Sci. 1258, 1-8 (2012).

4. Caldwell, J.M. et al. Cadherin 26 is an alpha integrin-binding epithelial receptor regulated during allergic inflammation. Mucosal Immunol. 10, 1190-1201 (2017).

5. Knight, D.A., Stick, S.M. \& Hackett, T.L. Defective function at the epithelial junction: a novel therapeutic frontier in asthma? J. Allergy. Clin. Immunol. 128, 557-558 (2011).

6. Xiao, C. et al. Defective epithelial barrier function in asthma. J. Allergy Clin. Immunol. 128, 549-556 (2011).

7. Hadley, G.A. \& Higgins, J.M. Integrin $\alpha E \beta 7$ : molecular features and functional significance in the immune system. Adv. Exp. Med. Biol. 819, 97-110 (2014).

8. Lamb, C.A. et al. $\alpha E \beta 7$ integrin identifies subsets of pro-inflammatory colonic $\mathrm{CD} 4^{+} \mathrm{T}$ lymphocytes in ulcerative colitis. J Crohns Colitis 11, 610-620 (2017).

9. Blanchard, C. et al. IL-13 involvement in eosinophilic esophagitis: transcriptome analysis and reversibility with glucocorticoids. J. Allergy Clin. Immunol. 120, 1292-1300 (2017).

10. Frøslie, K.F., Jahnsen, J., Moum, B.A., Vatn M.H.; IBSEN Group. Mucosal healing in inflammatory bowel disease: results from a Norwegian population-based cohort. Gastroenterology 133 , 412-422 (2007). 